DOI: $10.17516 / 1997-1397-2021-14-3-376-388$

УДК 517.95

\title{
The Time-fractional Airy Equation on the Metric Graph
}

\author{
Kamoladdin Rakhimov* \\ National University of Uzbekistan \\ Tashkent, Uzbekistan \\ Zarifboy Sobirov ${ }^{\dagger}$ \\ University of Geological Sciences \\ Tashkent, Uzbekistan \\ Nasridin Jabborov ${ }^{\ddagger}$ \\ National University of Uzbekistan \\ Tashkent, Uzbekistan
}

Received 10.09.2020, received in revised form 10.12.2020, accepted 20.02.2021

\begin{abstract}
Initial boundary value problem for the time-fractional Airy equation on a graph with finite bonds is considered in the paper. Properties of potentials for this equation are studied. Using these properties the solutions of the considered problem were found. The uniqueness theorem is proved using the analogue of Grönwall-Bellman inequality and a-priory estimate.
\end{abstract}

Keywords: time-fractional Airy equation, IBVP, PDE on metric graphs, fundamental solutions, integral representation.

Citation: K. Rakhimov, Z. Sobirov, N. Jabborov, The Time-fractional Airy Equation on the Metric Graph, J. Sib. Fed. Univ. Math. Phys., 2021, 14(3), 376-388.

DOI: 10.17516/1997-1397-2021-14-3-376-388.

\section{Introduction}

In recent years noticeable interest has been shown in the study of initial and initial-boundary value problems for equations of fractional order. This is due to the fact that fractional-integral calculus have applications in the study of diffusion and dispersion processes in various fields of science (see [1-5]).

The Schrodinger equation on metric graphs was studied (see $[6,7]$ and references therein). Such graphs sometimes called quantum graphs. The Schrodinger equation on the metric graph was also studied with Fokas unified transformation method [8].

The Airy equation on an interval was studied with Fokas unified transform method [9] and [10]. The potential theory for solutions of this equation was developed [11] and [12]. The linearised Airy equation on metric graphs was considered in [13-16] and [17]. M. Cavalcante considered non linearised KdV equation [18].

A. Pskhu studied properties of the Airy equation with time-fractional derivative. Fundamental solution of the equation was found and properties of potentials were studied (see [19]). Later, second fundamental solution was found and the properties of the some additional potentials were

*kamoliddin ru@inbox.ru

†sobirovzar@gmail.com

†jabborov61@mail.ru

(c) Siberian Federal University. All rights reserved 
studied [20,21]. Using this results solutions of initial and some IBVPs over infinite and finite intervals were found.

In this paper we consider the initial boundary value problem (IBVP) on a closed star graph with finite bonds. The solutions are found with the use of the potential method developed in [19-21].

\section{Basic concepts}

The operator

$$
{ }_{C} D_{\eta, t}^{\alpha} g(t)=\frac{1}{\Gamma(1-\alpha)} \int_{\eta}^{t} \frac{g^{\prime}(\xi)}{|t-\xi|^{\alpha}} d \xi, \quad 0<\alpha<1,
$$

is called fractional derivative (Caputo derivative) (see [22]), where $\Gamma(x)$ is the Gamma function. Inverse of this operator is called operator of fractional integration

$$
J_{\eta, t}^{\alpha} g(t)=\frac{1}{\Gamma(\alpha)} \int_{\eta}^{t} \frac{g(\xi)}{|t-\xi|^{1-\alpha}} d \xi
$$

It is easy to show that

$$
{ }_{C} D_{\eta, t}^{\alpha} g(t)={ }_{C} D_{0, t-\eta}^{\alpha} g(t)
$$

Function

$$
\phi(\lambda, \mu ; z):=\sum_{n=0}^{\infty} \frac{z^{n}}{n ! \Gamma(\lambda n+\mu)}, \quad \lambda>-1, \mu \in \mathrm{C}
$$

is called Wright function (see [23]). Wright function can be represented as

$$
\phi(\lambda, \mu ; z)=\frac{1}{2 \pi i} \int_{H a} e^{\sigma+z \sigma^{-\lambda}} \frac{d \sigma}{\sigma^{\mu}},
$$

where the integral is taken along the Hankel contour (see [22]). We have following estimate (see [19])

$$
|\phi(-\lambda, \mu ; z)| \leqslant C \exp \left(-\nu|z|^{\frac{1}{1-\lambda}}\right), C=C(\lambda, \mu, \nu)
$$

where $\nu<(1-\lambda) \lambda^{\frac{\lambda}{1-\lambda}} \cos \frac{\pi-|\arg z|}{1-\lambda}, \frac{1+\lambda}{2} \pi<|\arg z| \leqslant \pi$. The value of integral of this function is (see [19])

$$
\int_{0}^{+\infty} \phi(-\lambda, \mu ; a z) d z=-\frac{1}{a \Gamma(\mu+\lambda)}
$$

\section{Formulation of the problem}

The Cauchy problem for time-fractional Airy equation on a metric graph with infinite bonds was considered $([21])$. Now we consider a graph with $k$ incoming and $m$ outgoing bonds. In the incoming bonds coordinates are set from $L_{j}\left(L_{j}<0, j=\overline{1, k}\right)$ to 0 , and on the outgoing bonds the coordinates are set from 0 to $L_{i}\left(L_{i}>0, i=\overline{k+1, k+m}\right)$. The bonds of the graph are denoted by $b_{j}, j=\overline{1, k+m}$ (Fig. 1 ).

On each bond $b_{j}(j=\overline{1, k+m})$ of the graph, we consider the Airy equation with a fractional time derivative

$$
{ }_{C} D_{0, t}^{\alpha} u_{j}(x, t)-\frac{\partial^{3}}{\partial x^{3}} u_{j}(x, t)=f_{j}(x, t), \quad 0<t \leqslant T
$$




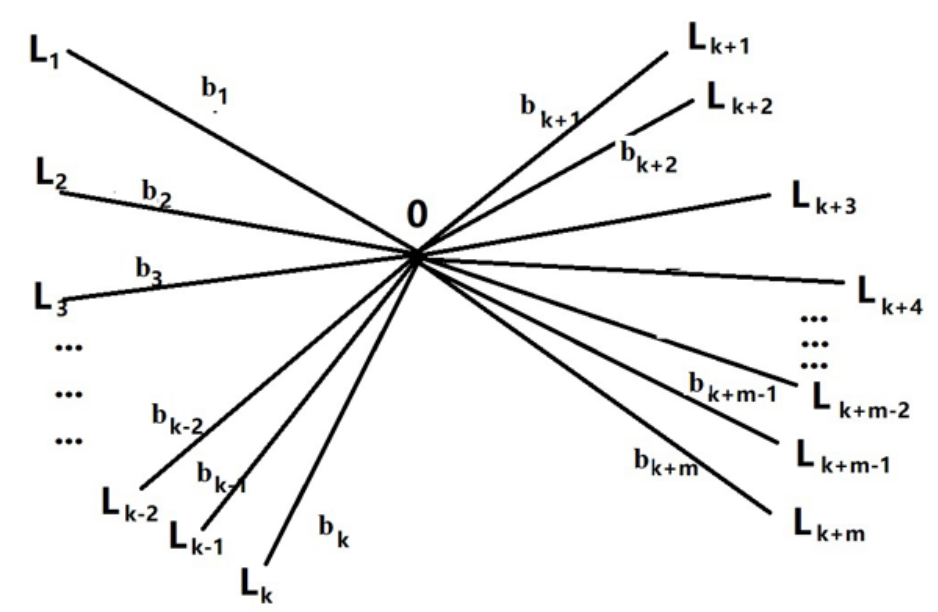

Fig. 1. Star-shaped graph

Let $0 \leqslant t \leqslant T$, and $x \in \overline{b_{j}}, j=\overline{1, k+m}$. We need to impose the following initial conditions

$$
u(x, 0)=u_{0}(x)
$$

vertex conditions

$$
\begin{gathered}
A u(0, t)=0, \\
\frac{\partial}{\partial x} u^{+}(0, t)=B \frac{\partial}{\partial x} u^{-}(0, t),
\end{gathered}
$$

where $u^{-}=\left(u_{1}, u_{2}, \ldots, u_{k}\right)^{T}, u^{+}=\left(u_{k+1}, u_{k+2}, \ldots, u_{k+m}\right)^{T}, u=\left(\begin{array}{c}u^{+} \\ u^{-}\end{array}\right)$,

$$
A=\left(\begin{array}{ccccc}
1 & -a_{2} & 0 & \ldots & 0 \\
1 & 0 & a_{3} & \ldots & 0 \\
\ldots & \ldots & \ldots & \ldots & \ldots \\
1 & 0 & 0 & \ldots & 0 \\
1 & 0 & 0 & \ldots & -a_{k+m}
\end{array}\right)
$$

and $B$ is the constant $m$-by- $k$ matrix.

We need impose the following conditions which are sometimes called the Kirchhoff conditions or the condition of conservation of flow rate at the vertex of the graph

$$
\left.C^{-} \frac{\partial^{2} u^{-}(x, t)}{\partial x^{2}}\right|_{x=0}=\left.C^{+} \frac{\partial^{2} u^{+}(x, t)}{\partial x^{2}}\right|_{x=0}
$$

where $C^{-}=\left(\frac{1}{a_{1}}, \frac{1}{a_{2}}, \ldots, \frac{1}{a_{k}}\right), C^{+}=\left(\frac{1}{a_{k+1}}, \ldots, \frac{1}{a_{k+m}}\right), a_{1}=1$ and $a_{j} \neq 0$ for $j=\overline{2, k+m}$. Boundary conditions are

$$
u(L, t)=\varphi(t),\left.\quad \frac{\partial u^{-}(x, t)}{\partial x}\right|_{x=L^{-}}=\phi(t),
$$

where $\varphi=\left(\varphi_{1}, \varphi_{2}, \ldots, \varphi_{k+m}\right)^{T}$ and $\phi=\left(\phi_{1}, \phi_{2}, \ldots, \phi_{k}\right)^{T}$.

A regular solution of equation (7) is constructed on the graph defined above that satisfies conditions (8)-(12). 


\subsection{Uniqueness of solution}

Theorem 1. Let $B^{T} B-I_{k}$ be negative defined matrix. Then problem (7), (8)-(12) has at most one solution.

Proof. Let us consider the following inequality [24]

$$
\int_{a}^{b} v_{C} D_{0, t}^{\alpha} v d x \geqslant \frac{1}{2} C_{C, t}^{\alpha} \int_{a}^{b} v^{2} d x
$$

. Using the Cauchy inequality and conditions (8)-(9), we have

$$
{ }_{C} D_{0, t}^{\alpha}\|u\|_{0}^{2} \leqslant\left(u^{-}\right)^{T}\left(B^{T} B-I_{k}\right)\left(u^{-}\right)+2\|u\|_{0}\|f\|_{0} \leqslant 2\|u\|_{0}\|f\|_{0} \leqslant\|u\|_{0}^{2}+\|f\|_{0}^{2},
$$

where

$$
\|u\|_{0}^{2}=\sum_{j=1}^{k+m} \int_{B_{j}} u_{j}^{2} d x
$$

$u=\left(u_{1}, u_{2}, \ldots, u_{k+m}\right)$.

Using the analogue of Grönwall's inequality [24], we obtain from the last inequality the following a priori estimate

$$
\|u\|_{0}^{2} \leqslant\left\|u_{0}\right\|^{2} E_{\alpha}\left(2 t^{\alpha}\right)+\Gamma(\alpha) E_{\alpha, \alpha}\left(2 t^{\alpha}\right)_{C} D_{0, t}^{\alpha}\|f\|_{0}^{2} .
$$

The proof of the theorem follows from (13).

\subsection{Fundamental solutions}

We construct the solution of the problem with the use of the potential method. To begin with, we need to obtain a special solution of equation (7) that is called fundamental solution. A fundamental solution of the equation was found in the following form [19]

$$
G_{\alpha}^{2 \alpha / 3}(x, t)=\frac{1}{3 t^{1-2 \alpha / 3}} \begin{cases}\phi\left(-\alpha / 3,2 \alpha / 3 ; \frac{x}{t^{\alpha / 3}}\right), & x<0, \\ -2 \operatorname{Re}\left[e^{2 \pi i / 3} \phi\left(-\alpha / 3,2 \alpha / 3 ; e^{2 \pi i / 3} \frac{x}{t^{\alpha / 3}}\right)\right], & x>0 .\end{cases}
$$

Using results from [21], second fundamental solution can be written in the following form

$$
V_{\alpha}^{2 \alpha / 3}(x, t)=\frac{1}{3 t^{1-2 \alpha / 3}} \operatorname{Im}\left[e^{2 \pi i / 3} \phi\left(-\alpha / 3,2 \alpha / 3 ; e^{2 \pi i / 3} \frac{x}{t^{\alpha / 3}}\right)\right], \quad x>0 .
$$

These functions have the following properties (see [19])

$$
{ }_{C} D_{0, t}^{\nu} G_{\sigma}^{\mu}(x, t)=G_{\sigma}^{\mu-\nu}(x, t), \quad \frac{\partial^{3}}{\partial x^{3}} G_{\sigma}^{\mu}(x, t)=G_{\sigma}^{\mu-\sigma}(x, t)
$$

with estimate

$$
\left|{ }_{C} D_{0, t}^{\nu} G_{\sigma}^{\mu}(x, t)\right| \leqslant C x^{-\theta} t^{\mu+\theta \sigma / 3-1},
$$

where

$$
\theta \geqslant \begin{cases}0, & (-\mu) \notin \mathbf{N}_{0} \\ 1, & (-\mu) \in \mathbf{N}_{0}\end{cases}
$$


Using these functions we define functions that are called potentials

$$
\begin{gathered}
w_{1}(x, t)=\int_{0}^{t} G_{\alpha}^{2 \alpha / 3}(x-a, t-\eta) \tau_{1}(\eta) d \eta, \quad w_{2}(x, t)=\int_{0}^{t} V_{\alpha}^{2 \alpha / 3}(x-a, t-\eta) \tau_{2}(\eta) d \eta \\
w_{3}(x, t)=\int_{0}^{t} \frac{\partial^{2}}{\partial x^{2}} G_{\alpha^{2} \alpha / 3}(x-a, t-\eta) \tau_{3}(\eta) d \eta, \quad w_{4}(x, t)=\int_{0}^{t} \frac{\partial^{2}}{\partial x^{2}} V_{\alpha}^{2 \alpha / 3}(x-a, t-\eta) \tau_{4}(\eta) d \eta \\
w_{5}(x, t)=\int_{a}^{b} G_{\alpha}^{2 \alpha / 3}(x-\xi, t) \tau_{5}(\xi) d \xi \text { and } w_{6}(x, t)=\int_{0}^{t} \int_{a}^{b} G_{\alpha}^{2 \alpha / 3}(x-\xi, t-\eta) f(\xi, \eta) d \xi d \eta
\end{gathered}
$$

Let us show some properties of these functions in the following lemmas.

Lemma 1. Let functions $\tau_{k}(t), k=1,2$ are continuous and bounded on $(0 ;+\infty)$. Then

1. Functions $w_{1}(x, t)$ and $w_{2}(x, t)$ are solutions of the equation

$$
{ }_{C} D_{0, t}^{\alpha} u_{j}(x, t)-\frac{\partial^{3} u_{j}(x, t)}{\partial x^{3}}=0 ;
$$

2. Functions $w_{1}(x, t)$ and $w_{2}(x, t)$ satisfy conditions

$$
\lim _{t \rightarrow 0} w_{k}(x, t)=0, k=1,2 .
$$

Lemma 2. Let $\tau_{3}(\eta), \tau_{4}(\eta) \in C V L(0, h)$. Then

$$
\lim _{x \rightarrow a-0} w_{3}(x, t)=\frac{1}{3} \tau_{3}(t), \quad \lim _{x \rightarrow a+0} w_{3}(x, t)=-\frac{2}{3} \tau_{3}(t), \quad \lim _{x \rightarrow a+0} w_{4}(x, t)=0 .
$$

The proofs of these lemmas can be found in [21].

Lemma 3. Let $\tau_{5}(x) \in C[a, b]$. Then function $w_{5}(x, t)$ is the fundamental solution of equation (7) and

$$
\lim _{t \rightarrow 0} C_{0, t}^{\alpha-1} w_{5}(x, t)=\tau_{5}(x) .
$$

Proof. Let us show that function $w_{5}(x, t)$ is the fundamental solution of equation (7). Using relations (16), we obtain

$$
{ }_{C} D_{0, t}^{\alpha} w_{5}(x, t)=\int_{a}^{b}{ }_{C} D_{0, t}^{\alpha} G_{\alpha}^{2 \alpha / 3}(x-\xi, t) \tau_{5}(\xi) d \xi=\int_{a}^{b} G_{\alpha}^{-\alpha / 3}(x-\xi, t) \tau_{5}(\xi) d \xi
$$

and

$$
\frac{\partial^{3}}{\partial x^{3}} w_{5}(x, t)=\int_{a}^{b} \frac{\partial^{3}}{\partial x^{3}} G_{\alpha}^{2 \alpha / 3}(x-\xi, t) \tau_{5}(\xi) d \xi=\int_{a}^{b} G_{\alpha}^{-\alpha / 3}(x-\xi, t) \tau_{5}(\xi) d \xi .
$$

Comparing these equalities, we obtain that function $w_{5}(x, t)$ is the fundamental solution of equation (7).

Let us find

$$
{ }_{C} D_{0, t}^{\alpha-1} w_{5}(x, t)=\int_{a}^{b}{ }_{C} D_{0, t}^{\alpha-1} G_{\alpha}^{2 \alpha / 3}(x-\xi, t) \tau_{5}(\xi) d \xi=\int_{a}^{b} G_{\alpha}^{1-\alpha / 3}(x-\xi, t) \tau_{5}(\xi) d \xi .
$$

Using inequality (17), we have the following estimate

$$
\left|{ }_{C} D_{0, t}^{\alpha-1} w_{5}(x, t)\right|=\left|\int_{a}^{b} G_{\alpha}^{1-\alpha / 3}(x-\xi, t) \tau_{5}(\xi) d \xi\right| \leqslant\left|\max _{a \leqslant x \leqslant b} \tau_{5}(x) \int_{a}^{b} C\right| x-\left.\xi\right|^{-\theta} t^{(1-\theta) \frac{\alpha}{3}} d \xi \mid,
$$


where $1>\theta \geqslant 0$. It shows that the integral form converges. Replacing $\frac{x-\xi}{t^{\alpha / 3}}$ with $y$ and taking into account that

$$
\begin{gathered}
\int_{-\infty}^{\infty} g_{\alpha}(y) d y=\int_{-\infty}^{\infty} t^{\alpha / 3} G_{\alpha}^{1-\alpha / 3}\left(y t^{\alpha / 3}, t\right) d y= \\
=t^{\alpha / 3} \int_{-\infty}^{0} \frac{1}{3 t^{\alpha / 3}} \phi\left(-\frac{\alpha}{3}, 1-\frac{\alpha}{3} ; y\right) d y-2 t^{\alpha / 3} \operatorname{Re}\left[e^{2 \pi i / 3} \int_{0}^{\infty} \frac{1}{3 t^{\alpha / 3}} \phi\left(-\frac{\alpha}{3}, 1-\frac{\alpha}{3} ; e^{2 \pi i / 3} y\right) d y\right]= \\
=\frac{1}{3} \int_{-\infty}^{0} \phi\left(-\frac{\alpha}{3}, 1-\frac{\alpha}{3} ; y\right) d y-2 \operatorname{Re}\left[e^{2 \pi i / 3} \frac{1}{3} \int_{0}^{\infty} \phi\left(-\frac{\alpha}{3}, 1-\frac{\alpha}{3} ; e^{2 \pi i / 3} y\right) d y\right]= \\
=\frac{1}{3}\left(\frac{1}{\Gamma(1-\alpha / 3+\alpha / 3)}-2 \operatorname{Re}\left[-e^{2 \pi i / 3} \frac{1}{e^{2 \pi i / 3} \Gamma(1-\alpha / 3+\alpha / 3)}\right]\right)=1,
\end{gathered}
$$

we obtain

$$
\begin{gathered}
\lim _{t \rightarrow 0} C D_{0, t}^{\alpha-1} w_{5}(x, t)=\lim _{t \rightarrow 0} \int_{a}^{b} G_{\alpha}^{1-\alpha / 3}(x-\xi, t) \tau_{5}(\xi) d \xi= \\
=\lim _{t \rightarrow 0} \int_{\frac{x-b}{t^{\alpha / 3}}}^{\frac{x-a}{t^{\alpha / 3}}} t^{\alpha / 3} G_{\alpha}^{1-\alpha / 3}\left(y t^{\alpha / 3}, t\right) \tau_{5}\left(x-t^{\alpha / 3} y\right) d y=\frac{\tau_{5}(x)}{3} \int_{-\infty}^{+\infty} g_{\alpha}(y) d y=\tau_{5}(x) .
\end{gathered}
$$

The lemma is proved.

Lemma 4. The equation ${ }_{C} D_{0, t}^{\alpha} u(x, t)-\frac{\partial^{3}}{\partial x^{3}} u(x, t)=f(x, t)$ with initial condition

$$
\left.{ }_{C} D_{0, t}^{\alpha-1} u(x, t)\right|_{t=0}=0
$$

has a solution in the form

$$
w_{6}(x, t)=\int_{0}^{t} d \eta \int_{a}^{b} G_{\alpha}^{2 \alpha / 3}(x-\xi, t-\eta) f(\xi, \eta) d \xi .
$$

Proof. Using the results given in [19], it is easy to show that solution of the Cauchy problem for the homogeneous equation ${ }_{C} D_{0, t}^{\alpha} v(x, t)-\frac{\partial^{3}}{\partial x^{3}} v(x, t)=0$ with initial condition $v(x, 0)=v_{0}(x)$ is

$$
v(x, t)={ }_{C} D_{0, t}^{\alpha-1} \int_{a}^{b} G_{\alpha}^{2 \alpha / 3}(x-\xi, t) v_{0}(\xi) d \xi
$$

Let us determine the derivatives of function $w_{6}(x, t)$

$$
\begin{gathered}
{ }_{C} D_{0, t}^{\alpha} w_{6}(x, t)=\frac{d}{d t} \int_{0}^{t} d \eta \int_{a}^{b}{ }_{C} D_{\eta, t}^{\alpha-1} G_{\alpha}^{2 \alpha / 3}(x-\xi, t-\eta) f(\xi, \eta) d \xi= \\
=\lim _{\eta \rightarrow t} \int_{a}^{b}{ }_{C} D_{\eta, t}^{\alpha-1} G_{\alpha}^{2 \alpha / 3}(x-\xi, t-\eta) f(\xi, \eta) d \xi+\int_{0}^{t} d \eta \int_{a}^{b} \frac{d}{d t} C_{C} D_{\eta, t}^{\alpha-1} G_{\alpha}^{2 \alpha / 3}(x-\xi, t-\eta) f(\xi, \eta) d \xi
\end{gathered}
$$

Taking into account (3) and relation (16), we obtain

$$
\begin{gathered}
\int_{a}^{b}{ }_{C} D_{\eta, t}^{\alpha-1} G_{\alpha}^{2 \alpha / 3}(x-\xi, t-\eta) f(\xi, \eta) d \xi=\int_{a}^{b}{ }_{C} D_{0, t-\eta}^{\alpha-1} G_{\alpha}^{2 \alpha / 3}(x-\xi, t-\eta) f(\xi, \eta) d \xi= \\
=\int_{a}^{b} G_{\alpha}^{1-\alpha / 3}(x-\xi, t-\eta) f(\xi, \eta) d \xi .
\end{gathered}
$$


It follows from relation (5) that integral $I_{1}$ converges uniformly. Substituting $\frac{x-\xi}{(t-\eta)^{\alpha / 3}}$ for $y$ in this integral and taking into account (6), we obtain

$$
\begin{aligned}
& I_{1}=\lim _{\eta \rightarrow t} \int_{a}^{b}{ }_{C} D_{\eta, t}^{\alpha-1} G_{\alpha}^{2 \alpha / 3}(x-\xi, t-\eta) f(\xi, \eta) d \xi=\lim _{\eta \rightarrow t} \int_{a}^{b} G_{\alpha}^{1-\alpha / 3}(x-\xi, t-\eta) f(\xi, \eta) d \xi= \\
& =\lim _{\eta \rightarrow t} \int_{\frac{x-b}{(t-\eta)^{\alpha / 3}}}^{\frac{x-a}{(t-\eta)^{\alpha / 3}}} G_{\alpha}^{1-\alpha / 3}\left((t-\eta)^{\alpha / 3} y, t-\eta\right) f\left(x-(t-\eta)^{\alpha / 3} y, \eta\right)(t-\eta)^{\alpha / 3} d y= \\
& =\lim _{\eta \rightarrow t} \int_{0}^{\frac{x-a}{(t-\eta)^{\alpha / 3}}} \frac{-2 f\left(x-(t-\eta)^{\alpha / 3} y, \eta\right)(t-\eta)^{\alpha / 3}}{3(t-\eta)^{\alpha / 3}} \operatorname{Re}\left(e^{2 \pi i / 3} \phi\left(-\frac{\alpha}{3}, 1-\frac{\alpha}{3} ; e^{2 \pi i / 3} y\right)\right) d y+ \\
& +\lim _{\eta \rightarrow t} \int_{\frac{x-b}{(t-\eta)^{\alpha / 3}}}^{0} \frac{1}{3(t-\eta)^{\alpha / 3}} \phi\left(-\frac{\alpha}{3}, 1-\frac{\alpha}{3} ; y\right) f\left(x-(t-\eta)^{\alpha / 3} y, \eta\right)(t-\eta)^{\alpha / 3} d y= \\
& =-\frac{2}{3} \lim _{\eta \rightarrow t} \int_{0}^{\frac{x-a}{(t-\eta)^{\alpha / 3}}} \operatorname{Re}\left(e^{2 \pi i / 3} \phi\left(-\frac{\alpha}{3}, 1-\frac{\alpha}{3} ; e^{2 \pi i / 3} y\right)\right) f\left(x-(t-\eta)^{\alpha / 3} y, \eta\right) d y+ \\
& +\frac{1}{3} \lim _{\eta \rightarrow t} \int_{\frac{x-b}{(t-\eta)^{\alpha / 3}}}^{0} \phi\left(-\frac{\alpha}{3}, 1-\frac{\alpha}{3} ; y\right) f\left(x-(t-\eta)^{\alpha / 3} y, \eta\right) d y= \\
& =-\frac{2}{3} \operatorname{Re}\left(\int_{0}^{+\infty} e^{2 \pi i / 3} \phi\left(-\frac{\alpha}{3}, 1-\frac{\alpha}{3} ; e^{2 \pi i / 3} y\right) f(x, t) d y\right)+\frac{1}{3} \int_{-\infty}^{0} \phi\left(-\frac{\alpha}{3}, 1-\frac{\alpha}{3} ; y\right) f(x, t) d y= \\
& =-\frac{2}{3} \operatorname{Re}\left(-e^{2 \pi i / 3} \frac{1}{e^{2 \pi i / 3} \Gamma(1)}\right) f(x, t)+\frac{1}{3}\left(-\frac{1}{-\Gamma(1)}\right) f(x, t)=f(x, t) .
\end{aligned}
$$

Now we have $I_{1}=f(x, t)$. Furthermore we show that $I_{2}=\frac{\partial^{3}}{\partial x^{3}} u(x, t)$. We begin with

$$
\begin{aligned}
I_{2} & =\int_{0}^{t} d \eta \int_{a}^{b} \frac{d}{d t} C D_{\eta, t}^{\alpha-1} G_{\alpha}^{2 \alpha / 3}(x-\xi, t-\eta) f(\xi, \eta) d \xi= \\
& =\int_{0}^{t} d \eta \int_{a}^{b} \frac{d}{d t} C^{D_{0, t-\eta}^{\alpha-1}} G_{\alpha}^{2 \alpha / 3}(x-\xi, t-\eta) f(\xi, \eta) d \xi= \\
& =\int_{0}^{t} d \eta \int_{a}^{b} \frac{d}{d t} G_{\alpha}^{1-\alpha / 3}(x-\xi, t-\eta) f(\xi, \eta) d \xi .
\end{aligned}
$$

To determine $\frac{\partial^{3}}{\partial x^{3}} u(x, t)$ we use relation (16). So, we have

$$
\begin{gathered}
\frac{\partial^{3}}{\partial x^{3}} u(x, t)=\frac{\partial^{3}}{\partial x^{3}} \int_{0}^{t} d \eta \int_{a}^{b} G_{\alpha}^{2 \alpha / 3}(x-\xi, t-\eta) f(\xi, \eta) d \xi= \\
=\int_{0}^{t} d \eta \int_{a}^{b} \frac{\partial^{3}}{\partial x^{3}} G_{\alpha}^{2 \alpha / 3}(x-\xi, t-\eta) f(\xi, \eta) d \xi=\int_{0}^{t} d \eta \int_{a}^{b} G_{\alpha}^{2 \alpha / 3-\alpha}(x-\xi, t-\eta) f(\xi, \eta) d \xi= \\
=\int_{0}^{t} d \eta \int_{a}^{b} \frac{\partial^{3}}{\partial x^{3}} G_{\alpha}^{-\alpha / 3}(x-\xi, t-\eta) f(\xi, \eta) d \xi .
\end{gathered}
$$


Comparing (18) and (19), we obtain $I_{2}=\frac{\partial^{3}}{\partial x^{3}} u(x, t)$. The Lemma is proved.

\subsection{Existence of solutions}

Let

$$
\begin{aligned}
& F^{-}=\left(F_{1}, \ldots, F_{k}\right)^{T}, F^{+}=\left(F_{k+1}, \ldots, F_{k+m}\right)^{T}, \\
& \alpha^{-}=\left(\alpha_{1}, \ldots, \alpha_{k}\right)^{T}, \alpha^{+}=\left(\alpha_{k+1}, \ldots, \alpha_{k+m}\right)^{T}, \\
& \beta^{-}=\left(\beta_{1}, \ldots, \beta_{k}\right)^{T}, \beta^{+}=\left(\beta_{k+1}, \ldots, \beta_{k+m}\right)^{T}, \\
& \gamma^{-}=\left(\gamma_{1}, \ldots, \gamma_{k}\right)^{T}, \gamma^{+}=\left(\gamma_{k+1}, \ldots, \gamma_{k+m}\right)^{T}, \\
& \rho^{-}=\left(\rho_{1}, \rho_{2}, \ldots, \rho_{k}\right)^{T}, \rho^{+}=\left(\rho_{k+1}, \ldots, \rho_{k+m}\right)^{T}, \\
& \alpha=\left(\begin{array}{c}
\alpha^{-} \\
\alpha^{+}
\end{array}\right), \quad \beta=\left(\begin{array}{c}
\beta^{-} \\
\beta^{+}
\end{array}\right), \gamma=\left(\begin{array}{c}
\gamma^{-} \\
\gamma^{+}
\end{array}\right), \quad \rho=\left(\begin{array}{c}
\rho^{-} \\
\rho^{-}
\end{array}\right), \quad F=\left(\begin{array}{c}
F^{-} \\
F^{+}
\end{array}\right)
\end{aligned}
$$

and $b_{j}=\left\{\begin{array}{ll}\left(L_{j} ; 0\right), & j=\overline{1, k} \\ \left(0 ; L_{j}\right), & j=\overline{k+1, k+m}\end{array}\right.$.

Let us find solutions in the form

$$
\begin{gathered}
u_{j}(x, t)=\int_{0}^{t} G_{\alpha}^{2 \alpha / 3}\left(x-L_{j}, t-\tau\right) \alpha_{j}(\tau) d \tau+\int_{0}^{t} V_{\alpha}^{2 \alpha / 3}\left(x-L_{j}, t-\tau\right) \beta_{j}(\tau) d \tau+ \\
+\int_{0}^{t} G_{\alpha}^{2 \alpha / 3}(x-0, t-\tau) \gamma_{j}(\tau) d \tau+\int_{0}^{t} V_{\alpha}^{2 \alpha / 3}(x-0, t-\tau) \rho_{j}(\tau) d \tau+F_{j}(x, t), \quad j=\overline{1, k+m},
\end{gathered}
$$

where functions $\alpha_{j}, \gamma_{j}(j=\overline{1, k+m}), \beta_{j}(j=\overline{1, k}), \rho_{j}(j=\overline{k+1, k+m})$ are unknown functions, $\rho_{j}(t)=0,(j=\overline{1, k}) ; \beta_{i}(t)=0, i=\overline{k+1, k+m}$ and

$$
F_{j}(x, t)=\int_{b_{j}} u_{0, j}(\xi)_{C} D_{0, t}^{\alpha-1} G_{\alpha}^{2 \alpha / 3}(x-\xi, t-0) d \xi+\int_{0}^{t} \int_{b_{j}} G_{\alpha}^{2 \alpha / 3}(x-\xi, t-0) f_{j}(\xi, \tau) d \xi d \tau .
$$

It follows from Lemma 4 and the results given in [19] that these functions are the solutions of equation (7) and they satisfy initial conditions (8).

Taking into account condition (9), we have

$$
\begin{gathered}
a_{j} \int_{0}^{t} G_{\alpha}^{2 \alpha / 3}\left(-L_{j}, t-\tau\right) \alpha_{j}(\tau) d \tau+a_{j} \int_{0}^{t} V_{\alpha}^{2 \alpha / 3}\left(-L_{j}, t-\tau\right) \beta_{j}(\tau) d \tau+ \\
+a_{j} \int_{0}^{t} G_{\alpha}^{2 \alpha / 3}(0, t-\tau) \gamma_{j}(\tau) d \tau+a_{j} \int_{0}^{t} V_{\alpha}^{2 \alpha / 3}(0, t-\tau) \rho_{j}(\tau) d \tau+a_{j} F_{j}(0, t)= \\
=\int_{0}^{t} G_{\alpha}^{2 \alpha / 3}\left(-L_{1}, t-\tau\right) \alpha_{1}(\tau) d \tau+\int_{0}^{t} V_{\alpha}^{2 \alpha / 3}\left(-L_{1}, t-\tau\right) \beta_{1}(\tau) d \tau+ \\
+\int_{0}^{t} G_{\alpha}^{2 \alpha / 3}(0, t-\tau) \gamma_{1}(\tau) d \tau+F_{1}(0, t), \quad j=\overline{2, k+m} .
\end{gathered}
$$

Furthermore

$$
\int_{0}^{t}\left(G_{\alpha}^{2 \alpha / 3}\left(-L_{1}, t-\tau\right) \alpha_{1}(\tau)+V_{\alpha}^{2 \alpha / 3}\left(-L_{1}, t-\tau\right) \beta_{1}(\tau)\right) d \tau+
$$




$$
\begin{gathered}
+\int_{0}^{t} \frac{\phi\left(-\frac{\alpha}{3}, \frac{2 \alpha}{3} ; 0\right)}{3(t-\tau)^{1-2 \alpha / 3}} \gamma_{1}(\tau) d \tau+F_{1}(0, t)= \\
=a_{j} \int_{0}^{t} G_{\alpha}^{2 \alpha / 3}\left(-L_{j}, t-\tau\right) \alpha_{j}(\tau) d \tau+a_{j} \int_{0}^{t} V_{\alpha}^{2 \alpha / 3}\left(-L_{j}, t-\tau\right) \beta_{j}(\tau) d \tau+ \\
+a_{j} \int_{0}^{t} \frac{\phi\left(-\frac{\alpha}{3}, \frac{2 \alpha}{3} ; 0\right)}{3(t-\tau)^{1-2 \alpha / 3}} \gamma_{j}(\tau) d \tau+\operatorname{Im}\left[a_{j} \int_{0}^{t} \frac{e^{2 \pi i / 3} \phi\left(-\frac{\alpha}{3}, \frac{2 \alpha}{3} ; 0\right)}{3(t-\tau)^{1-2 \alpha / 3}} \rho_{j}(\tau) d \tau\right]+a_{j} F_{j}(0, t) .
\end{gathered}
$$

So, we have

$$
\begin{gathered}
a_{j} F_{j}(0, t)-F_{1}(0, t)=\int_{0}^{t} \frac{\frac{\sqrt{3} a_{j}}{2} \rho_{j}(\tau)-a_{j} \gamma_{j}(\tau)+\gamma_{1}(\tau)}{3 \Gamma\left(\frac{2 \alpha}{3}\right)(t-\tau)^{1-2 \alpha / 3}} d \tau- \\
-a_{j} \int_{0}^{t} G_{\alpha}^{2 \alpha / 3}\left(-L_{j}, t-\tau\right) \alpha_{j}(\tau) d \tau-a_{j} \int_{0}^{t} V_{\alpha}^{2 \alpha / 3}\left(-L_{j}, t-\tau\right) \beta_{j}(\tau) d \tau+ \\
+\int_{0}^{t} G_{\alpha}^{2 \alpha / 3}\left(-L_{1}, t-\tau\right) \alpha_{1}(\tau) d \tau+\int_{0}^{t} V_{\alpha}^{2 \alpha / 3}\left(-L_{1}, t-\tau\right) \beta_{1}(\tau) d \tau .
\end{gathered}
$$

and

$$
\begin{gathered}
\gamma_{1}(\tau)-a_{j} \gamma_{j}(\tau)+\frac{\sqrt{3} a_{j}}{2} \rho_{j}(\tau)=3_{C} D_{0, t}^{2 \alpha / 3}\left(a_{j} F_{j}(0, t)-F_{1}(0, t)\right)- \\
-3 a_{j C} D_{0, t}^{2 \alpha / 3}\left(\int_{0}^{t} G_{\alpha}^{2 \alpha / 3}\left(-L_{j}, t-\tau\right) \alpha_{j}(\tau) d \tau+\int_{0}^{t} V_{\alpha}^{2 \alpha / 3}\left(-L_{j}, t-\tau\right) \beta_{j}(\tau) d \tau\right)- \\
-3{ }_{C} D_{0, t}^{2 \alpha / 3}\left(\int_{0}^{t} G_{\alpha}^{2 \alpha / 3}\left(-L_{1}, t-\tau\right) \alpha_{1}(\tau) d \tau+\int_{0}^{t} V_{\alpha}^{2 \alpha / 3}\left(-L_{1}, t-\tau\right) \beta_{1}(\tau) d \tau\right) .
\end{gathered}
$$

From above relation we obtain

$$
\begin{gathered}
\gamma_{1}(\tau)-a_{j} \gamma_{j}(\tau)+\frac{\sqrt{3} a_{j}}{2} \rho_{j}(\tau)=3_{C} D_{0, t}^{2 \alpha / 3}\left(a_{j} F_{j}(0, t)-F_{1}(0, t)\right)- \\
-3\left(\int_{0}^{t} G_{\alpha}^{0}\left(-L_{1}, t-\tau\right) \alpha_{1}(\tau) d \tau+\int_{0}^{t} V_{\alpha}^{0}\left(-L_{1}, t-\tau\right) \beta_{1}(\tau) d \tau\right)+ \\
+3 a_{j}\left(\int_{0}^{t} G_{\alpha}^{0}\left(-L_{j}, t-\tau\right) \alpha_{j}(\tau) d \tau+\int_{0}^{t} V_{\alpha}^{0}\left(-L_{j}, t-\tau\right) \beta_{j}(\tau) d \tau\right), \quad j=\overline{2, k+m} .
\end{gathered}
$$

In a similar manner, we obtain from condition (10) that

$$
\begin{gathered}
B \gamma^{-}(t)-\gamma^{+}(t)+\frac{\sqrt{3}}{2} \rho^{+}(t)=3_{C} D_{0, t}^{\alpha / 3}\left(F_{x}^{+}(0, t)-B F_{x}^{-}(0, t)\right)- \\
-3 \int_{0}^{t}\left(B G_{\alpha}^{\alpha / 3}\left(L^{-}, t-\tau\right) \alpha^{-}(\tau)+B V_{\alpha}^{\alpha / 3}\left(-L^{-}, t-\tau\right) \beta^{-}(\tau)\right) d \tau+ \\
+3 \int_{0}^{t}\left(G_{\alpha}^{\alpha / 3}\left(-L^{+}, t-\tau\right) \alpha^{+}(\tau)\right) d \tau .
\end{gathered}
$$

Taking into account condition (11) and using Lemmas given above, we have

$$
\begin{gathered}
C^{-} \gamma^{-}(t)+2 C^{+} \gamma^{+}(t)=3 C \lim _{x \rightarrow 0} \frac{\partial^{2}}{\partial x^{2}} \int_{0}^{t} G_{\alpha}^{2 \alpha / 3}(x-L, t-\tau) \alpha(\tau) d \tau+ \\
+3 C \lim _{x \rightarrow 0} \frac{\partial^{2}}{\partial x^{2}} \int_{0}^{t} V_{\alpha}^{2 \alpha / 3}(x-L, t-\tau) \beta(\tau) d \tau+3 C F_{x x}(0, t),
\end{gathered}
$$


where $C=\left(-C^{-}, C^{+}\right)$.

Using conditions (12), we have

$$
\begin{aligned}
J_{0, t}^{2 \alpha / 3}\left(\alpha_{j}(t)+\frac{\sqrt{3}}{2} \beta_{j}(t)\right)+\int_{0}^{t} G_{\alpha}^{2 \alpha / 3}\left(L_{j}, t-\tau\right) \gamma_{j}(\tau) d \tau+ & \\
& \left.+\int_{0}^{t} V_{\alpha}^{2 \alpha / 3}\left(L_{j}, t-\tau\right) \rho_{j}(\tau) d \tau+F_{j}\left(L_{j}, t\right)=\varphi_{j}(t), \quad j=\overline{(} 1, k+m\right) .
\end{aligned}
$$

Applying the properties of fractional operators, we obtain

$$
\begin{gathered}
\alpha_{j}(t)+\frac{\sqrt{3}}{2} \beta_{j}(t)={ }_{C} D_{0, t}^{2 \alpha / 3}\left(\varphi_{j}(t)-F_{j}\left(L_{j}, t\right)\right)- \\
{ }_{-C} D_{0, t}^{2 \alpha / 3}\left(\int_{0}^{t} G_{\alpha}^{2 \alpha / 3}\left(L_{j}, t-\tau\right) \gamma_{j}(\tau) d \tau+\int_{0}^{t} V_{\alpha}^{2 \alpha / 3}\left(L_{j}, t-\tau\right) \rho_{j}(\tau) d \tau\right), \quad j=\overline{1, k+m}
\end{gathered}
$$

Equations given above can be written in the following form

$$
\begin{gathered}
\alpha(t)+\frac{\sqrt{3}}{2} \beta(t)=-\int_{0}^{t} G_{\alpha}^{0}(L, t-\tau) \gamma(\tau) d \tau-\int_{0}^{t} V_{\alpha}^{0}(L, t-\tau) \rho(\tau) d \tau+ \\
+{ }_{C} D_{0, t}^{2 \alpha / 3}(\varphi(t)-F(L, t)) .
\end{gathered}
$$

In a similar manner, we have from condition (12) that

$$
\begin{gathered}
\alpha^{-}(t)-\frac{\sqrt{3}}{2} \beta^{-}(t)=\int_{0}^{t} G_{\alpha}^{0}\left(L^{-}, t-\tau\right) \gamma^{-}(\tau) d \tau+\int_{0}^{t} V_{\alpha}^{0}\left(L^{-}, t-\tau\right) \rho^{-}(\tau) d \tau+ \\
+{ }_{C} D_{0, t}^{\alpha / 3}\left(\phi(t)-F_{x}^{-}\left(L^{-}, t\right)\right) .
\end{gathered}
$$

We obtain the following system of integral equations (20)-(24) with respect to unknowns $\Lambda(t)$

$$
Q \Lambda(t)+\int_{0}^{t} K(t-\tau) \Lambda(\tau) d \tau=H
$$

where $\Lambda$ is the unknown functions, $Q$ is the $(3 k+3 m)$-by- $(3 k+3 m)$ matrix, $K$ is the matrix of potentials. Using above system, the matrices can be written in the form

$$
H=\left(\begin{array}{c}
-3 A_{C} D_{0, t}^{2 \alpha / 3} F(0, t) \\
3_{C} D_{0, t}^{\alpha / 3}\left(F_{x}^{+}(0, t)-B F_{x}^{-}(0, t)\right) \\
3 C F_{x x}(0, t) \\
{ }_{C} D_{0, t}^{2 \alpha / 3}(\varphi(t)-F(L, t)) \\
{ }_{C} D_{0, t}^{\alpha / 3}\left(\phi(t)-F_{x}^{-}\left(L^{-}, t\right)\right)
\end{array}\right), \Lambda=\left(\begin{array}{c}
\alpha \\
\beta \\
\gamma \\
\rho
\end{array}\right), Q=\left(\begin{array}{cc}
0 & M \\
Q_{1} & 0
\end{array}\right)
$$

where $M$ is the matrix on the form

$$
M=\left(\begin{array}{ccc}
1_{(k-1) \times 1} & -\operatorname{diag}\left(a_{2}, \ldots, a_{k}\right) & 0 \\
1_{(m) \times 1} & -\operatorname{diag}\left(a_{k+1}, \ldots, a_{k+m}\right) & \frac{\sqrt{3}}{2} \operatorname{diag}\left(a_{k+1}, \ldots, a_{k+m}\right) \\
B & -I_{m} & \frac{\sqrt{3}}{2} I_{m} \\
\frac{1}{a_{1}} \ldots \frac{1}{a_{k}} & \frac{2}{a_{k+1}} \ldots \frac{2}{a_{k+m}} & 0 \\
-385- &
\end{array}\right),
$$




$$
\begin{gathered}
Q_{1}=\left(\begin{array}{ccc}
I_{k} & 0 & \frac{\sqrt{3}}{2} I_{k} \\
0 & I_{m} & 0 \\
I_{k} & 0 & -\frac{\sqrt{3}}{2} I_{k}
\end{array}\right) \text { and } K=\left(\begin{array}{cc}
K_{1} & 0 \\
0 & K_{2}
\end{array}\right) \text { where } \\
K_{1}=3\left(\begin{array}{cc}
-A G_{\alpha}^{0}(-L) & -A V_{\alpha}^{0}(-L) \\
C G_{\alpha}^{0}\left(L^{-}\right) \mid G_{\alpha}^{0}\left(-L^{+}\right) & V_{\alpha}^{0}\left(-L^{-}\right) \\
C \lim _{x \rightarrow 0} \frac{\partial^{2}}{\partial x^{2}} G_{\alpha}^{2 \alpha / 3}(x-L) & -C^{-} \lim _{x \rightarrow 0} \frac{\partial^{2}}{\partial x^{2}} V_{\alpha}^{2 \alpha / 3}(x-L)
\end{array}\right) \\
K_{2}=\left(\begin{array}{cc}
-G_{\alpha}^{0}(L) & -V_{\alpha}^{0}(L) \\
-G_{\alpha}^{0}\left(L^{-}\right) \mid 0 & V_{\alpha}^{0}\left(L^{+}\right)
\end{array}\right)
\end{gathered}
$$

It is obvious that $\operatorname{det}(Q) \neq 0$ and elements of matrix $K(t, \tau)$ are bounded and continuous functions on $(0, T)$. It was proved that $\operatorname{det} M \neq 0[21]$. So, matrix integral equation (25) has unique solution in $(C[0, t])^{2 k+m}$.

So, we arrive at the following theorem.

Theorem 2. Let $B^{T} B-I_{k}$ be negative defined matrix, functions $u_{j, 0}(x) \in C\left(\bar{b}_{j}\right), f_{j}(x, t) \in$ $C^{0,1}\left(\bar{b}_{j} \times[0, T]\right)$ for $j=\overline{1, k+m}, \varphi(t)$ and $\phi(t)$ are differentiable functions on $[0, T]$. Then problem (7)-(12) has unique solution on $0 \leqslant t \leqslant T$.

\section{References}

[1] A.A.Kilbas, H.M.Srivastava, Trujillo Theory and Applications of Fractional Differential Equations, North-Holland Mathematics Studies, Vol. 204, Amsterdam, etc., Elsevier, 2006.

[2] A.Carpintery, F.Mainardi (Eds.), Fractals and Fractional Calculus in Continuum Mechanics, CIAM Cources and Lectures, Vol. 376, Wien, Springer, 1997.

[3] R.Hilfer (Ed.), Applications of Fractional Calculus in Physics, Singapore, WSPC, 2000.

[4] R.Metzler, J.Klafter, The random walk's guide to anomalous diffusion: a fractional dynamics approach, Phys. Reports, 339(2000), 1-77.

[5] Y.S.Kivshar, G.P.Agarwal, Optical Solitons: From Fibers to Photonic Crystals, Academic, San Diego, 2003.

[6] T.Kottos, U.Smilansky, Periodic Orbit Theory and Spectral Statistics for Quantum Graphs, Annals of Physics, 274(1999), no. 1, 76-124.

[7] S.Gnutzmann, U.Smilansky, Quantum graphs: Applications to quantum chaos and universal spectral statistics, Advances in Physics, 55(2006), no. 5-6, 527-625.

[8] G.Khudayberganov, Z.Sobirov, M.Eshimbetov, Unified transform method for the Schrodinger equation on a simple metric graph, Journal of Siberian Federal University. Mathematics and Physics, 12(2019), no. 4, 412-420.

DOI: 10.17516/1997-1397-2019-12-4-412-420 
[9] B.Pelloni, Well-posed boundary value problems for linear evolution equations on a finite interval, Mathematical Proceedings of the Cambridge Philosophical Society, 136(2004), $361-382$.

[10] A.A.Himonas, D.Mantzavinos, F.Yan, The Korteweg-de Vries equation on an interval, Journal of Mathematical Physics, 60(2019), no. 5. DOI: 10.1063/1.5080366

[11] T.Jurayev, Boundary value problems for equations of mixed and mixed-composite types, "Fan", Tashkent, Uzbekistan, 1979 (in Russian).

[12] L.Cattabriga, Un problema al contorno per una equazione parabolica di ordine dispari, Annali della Scuola Normale Superiore di Pisa, 13(1959), no. 3, 163-203 .

[13] Z.A.Sobirov, H.Uecker, M.I.Akhmedov, Exact solutions of the Cauchy problem for the linearized KdV equation on metric star graphs, Uzbek Mathematical Journal, 3(2015), 143-154.

[14] Z.A.Sobirov, M.I.Akhmedov, U H.ecker, Cauchy problem for the linearized KdV equation on general metric star graphs, Nanosystems: Physics, Chemistry, Mathematics, 6(2015), no. $2,198-204$.

[15] D.Mugnolo, D.Noja, Ch.Seifert, Airy-type evolution equations on star graphs, Analysis and PDE, 11(2018), no. 7, 1625-1652. DOI: 10.2140/apde.2018.11.1625

[16] Z.A.Sobirov, M. I.Akhmedov, O.V.Karpova, B.Jabbarova, Linearized KdV equation on a metric graph, Nanosystems: Physics, Chemistry, Mathematics, 6(2015), no. 6, 757-761.

[17] Ch.Seifert, The linearized Korteweg-de-Vries equation on general metric graphs, The Diversity and Beauty of Applied Operator Theory, (2018), 449-458.

[18] M.Cavalcante, The Korteweg-de Vries equation on a metric star graph, Zeitschrift fur angewandte Mathematik und Physik, 69:124(2018). DOI: 10.1007/s00033-018-1018-6

[19] A.V.Pskhu, Fundamental solution of a third-order equation with a fractional derivative, Uzbek mathematical journal, 4(2017), 119-127 (in Russian).

[20] K.U.Rakhimov, The method of potentials for the Airy equation of fractional order, Bulletin of National University of Uzbekistan: Mathematics and Natural Sciences, 3(2020), no. 2, 222-235.

[21] Z.A.Sobirov, K.U.Rakhimov, Cauchy problem for the Airy equation with fractional timefractional on a star-shaped graph, Institute of Mathematics Bulletin. Uzbekistan, 5(2019), $40-49$.

[22] A.V.Pskhu, Fractional partial differential equations, Moscow, Russia, 2005.

[23] F.Mainardi, A.Mura, G.Pagnini, The M-Wright function in time-fractional diffusion processes: a tutorial survey, International Journal of Differential Equations, 3(2010).

[24] A.A.Alikhanov, A Priori Estimates for Solutions of Boundary Value Problems for FractionalOrder Equations, Differential equations, 46(2010), no. 5, 658-664. 


\title{
Уравнение Эйри с дробной производной по времени на метрическом графе
}

\author{
Камоладдин Рахимов \\ Национальный университет Узбекистана \\ Ташкент, Узбекистан \\ Зарифбой Собиров \\ Университет геологических наук \\ Ташкент, Узбекистан \\ Насридин Жабборов \\ Национальный университет Узбекистана \\ Ташкент, Узбекистан
}

\begin{abstract}
Аннотация. Мы рассматриваем задачу Коши и начально-краевую задачу для уравнении Эйри с дробной производной по времени на метрическом графе с ограниченными и с неограниченными ветвями. Мы изучали свойства потенциалов для этого уравнения и, используя эти свойства, нашли решения рассматриваемой задачи. Теорема единственности была доказана с помощью аналога неравенства Гронуолла-Беллмана и априорной оценки.
\end{abstract}

Ключевые слова: уравнение Эйри с дробной производной по времени, начально-краевая задача, уравнения в частных производных на метрическом графе, фундаментальные решения, интегральное представление. 